\title{
SENSITIVE MICROMIXER FOR DETECTING LOW CONCENTRATIONS OF IBUPROFEN
}

\author{
${ }^{1}$ Vasilii BURTSEV, ${ }^{1,2}$ Elena MILIUTINA, ${ }^{1,2}$ Mariia ERZINA, ${ }^{1}$ Vaclav ŠVORČíK, \\ 1,2Oleksiy LYUTAKOV
}

1 University of Chemistry and Technology, Prague, Czech Republic, EU, Iyutakoo@vscht.cz

${ }^{2}$ University of Strathclyde, Faculty of Physics, Tomsk, Russian Federation

https://doi.org/10.37904/nanocon.2019.8624

\begin{abstract}
In recent years, scientific research has been associated with diminishment the size of analytical tools with simultaneous improving their characteristics. In this regard, one of the most perspective areas is the microfluidic-based analytical platform. The utilization of microfluidic approach enables the analysis of small volumes of fluids (micro- and nanoliters). In addition, the microfluidic makes it possible to develop a new class of equipment - the so-called complex laboratory (Lab-on-a-chip) devices that allow analyzing complex analytes in various mixtures. In present work, the microfluidic-based micromixer with incorporated AuMs (gold multibranched NPs) nanoparticles with surface grafted oleophilic chemical moieties for detection of pharmaceutics with low solubility in water (ibuprofen has been used as a model compound due to the fact that this is a common pharmaceutics contaminant) was demonstrated. The proposed microfluidic device utilizes the unique advantages of microfluidic, which explores the unusual behavior of liquids limited by microscale and Surface-Enhanced Raman (SERS), capable of recognizing one molecule of the target analyte. The proposed approach is especially relevant in medical diagnostics to determine the drug digestibility and environment monitoring and protection areas.
\end{abstract}

Keywords: Lab-on-a-chip, SERS, 3D printing, microfluidics system, micromixer, ibuprofen

\section{INTRODUCTION}

Today microfluidic is one of the most relevant and rapidly developing areas of science and technology [1-4]. The microfluidic approach can reduce the size of all kinds of analytical laboratory devices and improve their technological characteristics [5,6]. Moreover, in combination with Surface-enhanced Raman spectroscopy (SERS), microfluidic devices can detect extremely low concentrations of the targeted analytes. In this work, we present a SERS/microfluidic-based approach, which allows detecting low concentrations of targeted analyte - ibuprofen. The ibuprofen has taken into account and has chosen for its wide medical utilization and related requiring of its detection in biofluids (drug digestibility estimation) or wastewater analysis [7-9].

\section{EXPERIMENTAL}

\subsection{Materials}

3D microfluidic micromixer was formed with polymer Clear resin (purchased from Formlabs, USA). Isopropyl alcohol ( $\geq 98.5 \%)$, deionized water, toluene $(99 \%)$, Ibuprofen were purchased from Sigma-Aldrich and used without further purification. The ADT- $\mathrm{C}_{16} \mathrm{H}_{33}$ salt was prepared by the procedure reported in $[10,11]$.

\subsection{Sample preparation}

\section{Microfluidic model preparation}

The microfluidic micromixer was made using 3D printing (the model is shown in Figure 2C). The model for printing was designed by using the program COMSOL Multiphysics. After fabrication, the microfluidic 
micromixer was additionally irradiated by UV-source for $30 \mathrm{~min}$, watched with isopropyl and dried at $40^{\circ} \mathrm{C}$ for $12 \mathrm{~h}$.

\section{Description of AuMs preparation}

Gold multibranched nanoparticles (AuMs) were synthesized using the procedure.[12]: $100 \mu \mathrm{L}$ of $10 \mathrm{mM}$

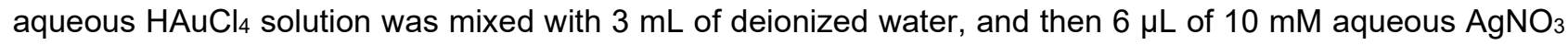
solution was added under magnetic stirring for $30 \mathrm{~s}$. After the solutions had been thoroughly mixed, $2 \mu \mathrm{L}$ of $100 \mathrm{mM}$ ascorbic acid was "quickly" added, and the solution was stirred vigorously for $10 \mathrm{~s}$ at room temperature (RT). When the reducing agent was added, the color of the mixture changed immediately from yellow to dark blue, indicating the formation of AuMs. Then the AuMs were dispersed in $3 \mathrm{~mL}$ of water and were purified three times by centrifugation at $5000 \mathrm{rpm}$ for $10 \mathrm{~min}$ [13]. For further use, the resulted precipitates were redisclosed in deionized water.

\section{Diazonium modification AuMs}

The obtained nanoparticles were spontaneously modified by soaking in $6 \mathrm{mM}$ freshly prepared aqueous solution of $A D T-\mathrm{C}_{16} \mathrm{H}_{33}$, in advance prepared according to the procedure described in [10,11] for $45 \mathrm{~min}$. After modification, the nanoparticles were rinsed with water, methanol, and acetone and dried at room conditions. The procedure of AuMs rinsing/drying was repeated three times. Finally, the grafted AuMs were dispersed in the toluene.

\section{Raman spectroscopy investigations- concentration dependence}

Raman data were acquired in the microfluidic chip at room temperature, using the customized microfluidic device equipped with microscopic Raman spectrometer ProRaman-L (785 nm excitation laser). The excitation power was $0.6 \mathrm{~mW}$ and the measurement time was $10 \mathrm{~s}$ with 60 times average. The presented Raman spectra were obtained by subtracting the toluene spectrum from the raw spectra.

Schematically the proposed microfluidic SERS (Surface-enhanced Raman spectroscopy) platform chip is demonstrated in the Figure 1.

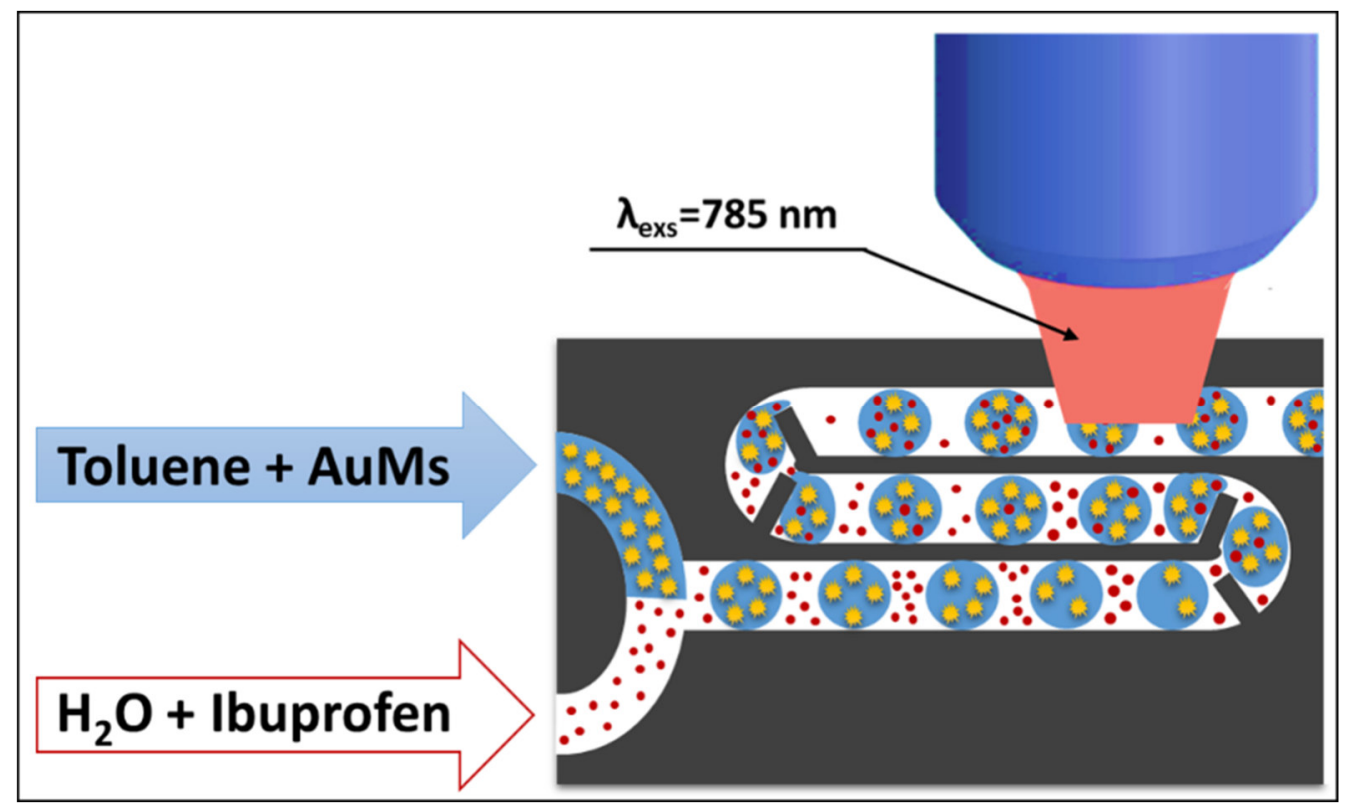

Figure 1 Graphical depiction of microfluidic micromixer and schematic of the concept of SERS measurement 


\section{RESULTS AND DISCUSSION}
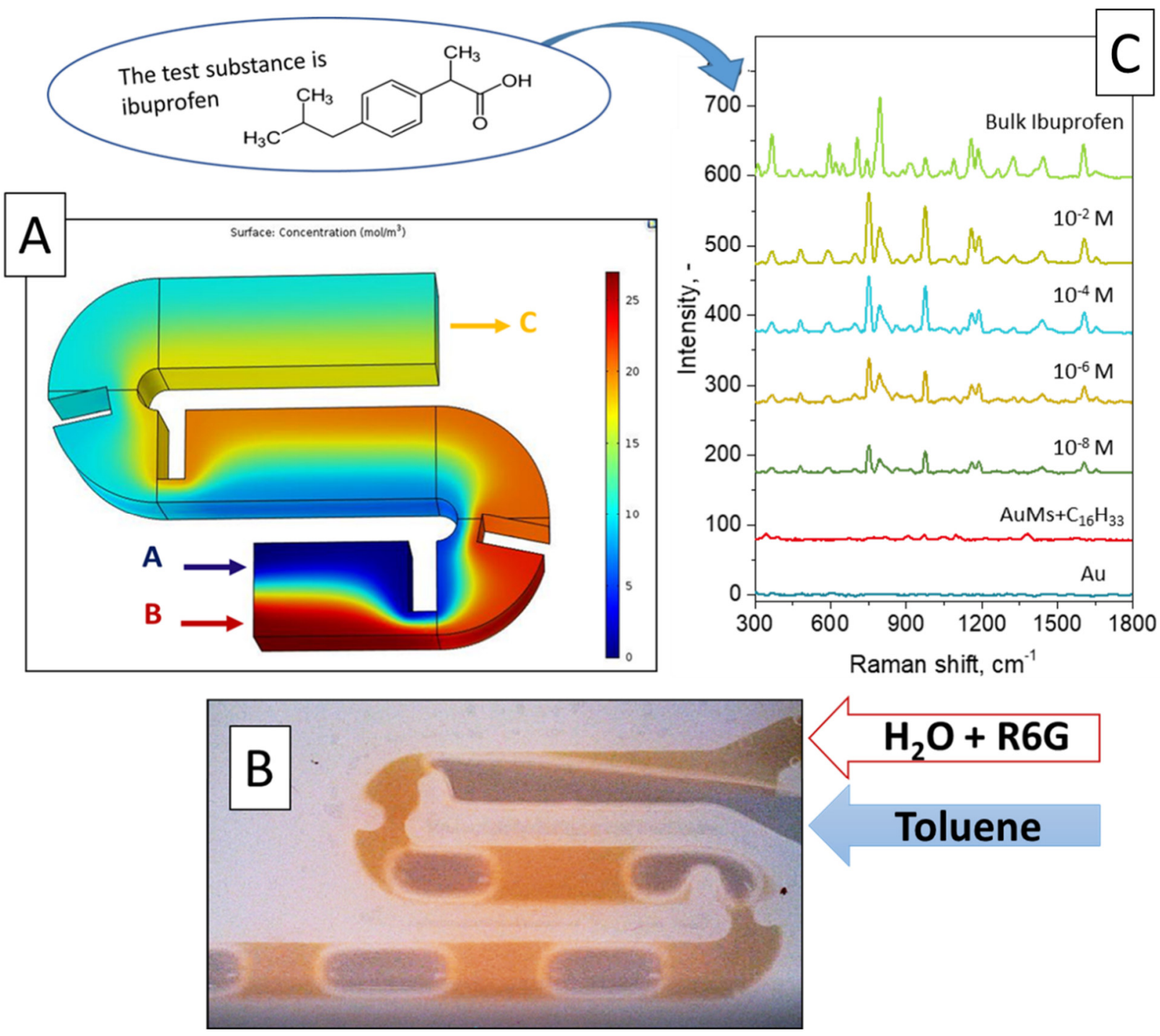

Figure 2 Figure $2 \mathrm{~A}$ - The mathematical model of a fluid mix in a micromixer, calculated with Comsol Multiphysics software. B - Photo of a real microfluidic sample. C - SERS spectra measured on bulk ibuprofen and concentration dependent ibuprofen (after baseline correction - subtraction of toluene response), measured in the microfluidic regime.

The well-known problem in the field of microfluidics has solved in the work - the thinner the channel, the greater role the viscous resistance of the liquid plays. Thus, the thinner the channel, the worse liquids are mixed since the flow is laminar. The developed model solves the problem of micromixing of liquid, as shown in Figure 2A. It is also known that Brownian particles (for example, nanoparticles spread very slowly over the channel crosssection. The only process that causes such particles to mix is diffusion. Unfortunately, the diffusion process is rather slow and cannot be used in modern analytical devices. Therefore, the passive (i.e. without moving parts) micromixer, which would effectively mix a suspension of nanoparticles and a solution with an analyte was developed (schematic representation $n$ is given in the Figure 2A).

In this work, the microfluidic model of a micromixer incorporating AuMs nanoparticles with oleophilic properties in a solution for SERS-based identification of poorly water-soluble drugs (ibuprofen was used as an example) 
was demonstrated (Figure 2B). The detection circuit includes two fluid streams mixing in a developed micromixer. The first stream is an aqueous solution containing various concentrations of ibuprofen. Toluene was chosen for the second stream because it mixes poorly with water but dissolves ibuprofen well. AuMs nanoparticles were prepared separately and their surface was grafted by ADT- $\mathrm{C}_{16} \mathrm{H}_{33}$ for increasing the stability of AuMs in toluene. The grafted AuMs were suspended in toluene and used as SERS probe. The system was designed in such a way that the ibuprofen with stirring gets into a solution of toluene containing nanoparticles and thus increase the SERS signal.

In order to present the work of our model more clearly, the water in the first stream was tinted with rhodamine 6G. As can be seen from Figure 2B, toluene does not mix with water, but small droplets form successfully flow through the microfluidic channel.

Raman data were acquired in the microfluidic chip at room temperature, using a customized microscopic Raman spectrometer equipped with a $785 \mathrm{~nm}$ excitation laser. During the test, the ibuprofen in water solution and AuMs nanoparticles in toluene were injected into the microchannel using syringe pumps. Raman spectra show that ibuprofen was detected in different concentrations $10^{-2} \mathrm{M}, 10^{-4} \mathrm{M}, 10^{-6} \mathrm{M}, 10^{-8} \mathrm{M}$ as shown in the Figure 2C. As the concentration of ibuprofen decreases, the intensities of the peaks of Raman scattering gradually decrease and almost disappear at a concentration below $10^{-8} \mathrm{~mol} \cdot \mathrm{L}^{-1}$. In conclusion, our approach allows us to extract and detect low concentrations of poorly water-soluble drugs.

\section{CONCLUSION}

In this work, the novel design of microfluidic-based micromixer chip for SERS detection of ibuprofen was presented. The microfluidic chip was designed in Comsol and fabricated using 3D printing. Numerical simulations were used for the optimization of micromixers structure for achieving the extraction of ibuprofen from water by the toluene phase. The AuMs modified with the aliphatic diazonium salt were added to the toluene and served as a SERS probe. In conclusion, our approach allows us to detect concentrations up to $10^{-8} \mathrm{~mol} \cdot \mathrm{L}^{-1}$ of poorly water-soluble drugs and this approach was demonstrated by using ibuprofen.

\section{ACKNOWLEDGEMENTS}

This work was supported by under the project IGA 1260881909.

\section{REFERENCES}

[1] FOUDEH, Amir, DIDAR, Tohid F., VERES, Teodor, TABRIZIAN, Maryam. Microfluidic Designs and Techniques Using Lab-on-a-Chip Devices for Pathogen Detection for Point-of-Care Diagnostics. Lab on a Chip. 2012. Issue 12, pp. 3249-3266.

[2] LAWANSTIEND, Duangtip, GATEMALA, Harchana, NOOTCHANAT, Supeera, EAKASIT, Sanong, WONGRAVEE, Kanet, SRISA-ART, Monpichar. Microfluidic approach for in situ synthesis of nanoporous silver microstructures as on-chip SERS substrates. Sensors and Actuators B: Chemical. 2018. 270, pp. 466-474.

[3] XIE, Jun, MIAO, Yunan, SHIH, Jason, TAI, Yu-Chong, LEE, Terry D. Microfluidic Platform for Liquid Chromatography-Tandem Mass Spectrometry Analyses of Complex Peptide Mixtures. Anal. Chem. 2005, 77, pp. 6947-6953.

[4] ASHOK, Praveen C., SINGH, Gajendra P., RENDALL, Helen A., KRAUSS, Thomas F., DHOLAKIA, Kishan. Waveguide confined Raman spectroscopy for microfluidic interrogation. Lab on a Chip. 2011. 11(7), pp. 12621270.

[5] HAEBERLE, Stefan, ZENGERLE, Roland. Microfluidic platforms for lab-on-a-chip applications. Lab on a Chip. 2007. 7(9), pp. 1094-1110. 
[6] PALLAORO, Alessia; HOONEJANI, Mehran R.; BRAUN, Gary B.; MEINHART, Carl D.; MOSKOVITS, Martin. Rapid Identification by Surface-Enhanced Raman Spectroscopy of Cancer Cells at Low Concentrations Flowing in a Microfluidic Channel. ACS Nano. 2015. 9, pp. 4328-4336.

[7] DAVIES, Neal M. Clinical Pharmacokinetics of Ibuprofen, Clin Pharmacokinet. 1998. 34, pp. 101-154.

[8] RAINSFORD, K. D. Ibuprofen: pharmacology, efficacy and safety. Inflammopharm. 2009. 17, pp. $275-342$.

[9] ALBERT, Kenneth; S. Gernaat, Charlene M. Pharmacokinetics of Ibuprofen, The American Journal of Medicine. 1984. 77 , pp. 40-46.

[10] GUSELNIKOVA, Olga; POSTNIKOV, Pavel; KALACHYOVA, Yevgeniya; KOLSKA, Zdenka; LIBANSKY, Milan; ZIMA, Jiri; SVORCIK, Vaclav; LYUTAKOV, Oleksiy. Large-Scale, Ultrasensitive, Highly Reproducible and Reusable Smart SERS Platform Based on PNIPAm-Grafted Gold Grating. ChemNanoMat, 2017. 3, pp. $135-144$.

[11] GUSELNIKOVA, Olga; KALACHYOVA, Yevgeniya; HROBONOVA, Karolina; TRUSOVA, Marina; BAREK, Jiri; POSTNIKOV, Pavel; SVORCIK, Vaclav; LYUTAKOV, Oleksiy. SERS platform for detection of lipids and disease markers prepared using modification of plasmonic-active gold gratings by lipophilic moieties. Sens. Act. B: Chem. 2018. 265, pp. 182-192.

[12] CHENG, Liang-Chien; HUANG, Jing-Hong; CHEN, Hao Ming; LAl, Tsung-Ching; YANG, Kuang-Yu; LIU, Ru-Shi; HSIAO, Michael; CHEN, Chung-Hsuan; HER, Li-Jane; TSAI, Din Ping. J. Mater. Chem., 2012, 22, pp. 2244-2253.

[13] KALACHYOVA, Y.; OLSHTREM, A.; Guselnikova, O. A.; Postnikov, P. S.; Elashnikov, R.; ULBRICH, P.; RIMPELOVA, S.; Svorcik V. and LYUTAKOV, O. Synthesis, Characterization, and Antimicrobial Activity of Near-IR Photoactive Functionalized Gold Multibranched Nanoparticles. ChemistryOpen, 2017, 6, pp. 254-260. 